\title{
Iterative Multi-User Turbo-Code Receiver for DS-CDMA*
}

\author{
D. Stienstra and A. K. Khandani \\ Dept. of Elec. and Comp. Eng., University of Waterloo, Waterloo, Ont., N2L 3G1 \\ Email: stienstra@shannon.uwaterloo.ca, khandani@shannon.uwaterloo.ca
}

\begin{abstract}
We present methods for the multi-user interference cancelation in a Code Division Multiple Access (CDMA) system where Turbo Codes are utilized for Forward Error Correction (FEC). In the proposed methods, the individual users are decoded separately with the operation of iterative interference cancelation being mixed with the iterative decoding of Turbo Codes. This results in a modest increase in the overall complexity as compared to a conventional single user receiver utilizing Turbo Codes. Numerical results are presented showing that in the cases of practical interest for CDMA applications, the multiple access interference can be essentially removed at a reasonable level of complexity. These iterative decoders achieve a similar to better performance with a substantial reduction in the complexity as compared to similar previously known research works.
\end{abstract}

\section{Introduction}

The performance of a CDMA system can be greatly enhanced by jointly decoding all the users instead of decoding them separately as done in the conventional receiver. However, most of the proposed multi-user detectors in the literature are designed with no consideration to channel coding. Only recently there has been a focus on developing multiuser detectors in combination with various channel coding methods. In [3], Hagenauer proposes an interference cancelation receiver for a synchronous system based on viewing the CDMA channel as a block code. This result led to the proposal of more complex sub-optimal multi-user receivers for asynchronous systems in $[4,5,6,7]$.

Reed et al. [8, 9] also proposed an iterative multi-user decoder for synchronous CDMA systems. These decoders utilize both convolutional codes [8] and Turbo Codes [9]. In both cases, near single-user performance was achieved. However, the complexity in both decoders is still exponential with respect to the number of users. Reference [9] also proposes a lower complexity decoder which however results in a noticeable degradation in the performance (in the order of a dB).

Alexander, Grant and Reed [10] further extended [8] to the asynchronous CDMA system using convolutional

\footnotetext{
*This work has been supported by Communications and Information Technology Ontario (CITO), Natural Sciences and Engineering Research Council of Canada (NSERC), and Nortel Networks. A more detailed version of this work can be found in $[1,2]$.
}

codes. Their proposed iterative decoder views the concatenation of direct-sequence spreading with the asynchronous multiple-access channel as a special form of convolutional code, implementing the interference cancelation operation as part of the decoding. This decoder performed within a fraction of a $\mathrm{dB}$ of the single-use bound for a heavily loaded system at high signal to noise ratio values. Note that most of the iterative interference cancelation schemes based on considering the spreading and the channel coding operations as a concatenated coding scheme require a fairly high signal to noise ratio to perform close to the single user bound. However, this restriction on the admissible range of signal to noise ratio does not apply to the schemes proposed in this article. More recently, Wang et al. [11] and El Gamal et al. [12] have proposed more advanced iterative sub-optimal receivers using convolutional code.

This paper proposes sub-optimal iterative multi-user receivers for CDMA, utilizing Turbo Codes [13], over an AWGN channel. The iterative structure of these receivers is similar to the iterative structure utilized by Reed et al. [9] in the sense that the joint detection is combined with channel decoding. However, unlike [9], the proposed receivers use the individual Turbo decoders to perform the task of interference cancelation, while adding minimal complexity to the Turbo decoding. This difference yields a complexity that is polynomial in the number of users for the proposed schemes for a comparable or better performance in cases of practical interest as compared to [9] which has an exponential complexity. As already mentioned, reference [9] also discuisses a decoder with a reduced complexity, but in their case the price to pay in terms of the performance is noticeable (in the order of a $\mathrm{dB}$ ). Throughout the paper, we follow a system model and a set of assumptions similar to [9] to provide a benchmark for comparison.

\section{CDMA System Model}

The discrete-time CDMA system used in this paper assumes that the channel is both chip and symbol synchronous. Note that a similar model is used in [9] which is the benchmark for the comparison in this paper. Generalization to the asynchronous channel is possible by incorporating an appropriate model to compute the conditional probability distribution for the MAI term. The $K$ users each transmit $M$ source bits $b_{t}^{(k)} \in\{0,1\}$ per each block of data, where 
$k \in\{1, \ldots, K\}$ specifies the user and $t \in\{1, \ldots, M\}$ is the time index of the uncoded data. The source bits for each user are assumed to be i.i.d. and are independent of the other users. The uncoded bits $b_{t}^{(k)}$ are coded into $L=M / R$ symbols $d_{t}^{(k)} \in\{-1,+1\}$ per block, where $k \in\{1, \ldots, K\}$ specifies the user, $t \in\{1, \ldots, L\}$ is the time index of the coded block, and $R$ is the coding rate. The discrete-time spreading signal utilized by user $k$ to spread the coded symbols at symbol interval $t$ consists of $N$ chips, normalized to unit energy, and is denoted by $s_{t}^{(k)} \in\left\{\frac{-1}{\sqrt{N}}, \frac{1}{\sqrt{N}}\right\}^{N}$. The decoder is assumed to have knowledge of the spreading signals of all the users.

The matched filter outputs of the $K$ users can be expressed in vector form as,

$$
\mathbf{r}_{t}=\mathbf{H}_{t} \mathbf{d}_{t}+\mathbf{n}_{t}
$$

where $\mathbf{r}_{t}=\left(r_{t}^{(1)}, \ldots, r_{t}^{(K)}\right)^{\top},(\cdot)^{\top}$ denotes the transposition operation, is the received vector, $\mathbf{d}_{t}=$ $\left(d_{t}^{(1)}, \ldots, d_{t}^{(K)}\right)^{\top}$ is the coded data vector, and $\mathbf{H}_{t}$ is the $K \times K$ discrete-time cross-correlation matrix of the spreading signals. The noise samples $\mathbf{n}_{t}=\left(n_{t}^{(\mathbf{1})}, \ldots, n_{t}^{(K)}\right)^{\top}$, as a result of matched filtering, are correlated with autocorrelation matrix $E\left\{\mathbf{n}_{t} \mathbf{n}_{t}^{\top}\right\}=\mathbf{H}_{t} \sigma^{2}$, where $E\{\}$ denotes the statistical expectation. The sampled output of the matched filter for the $k^{\text {th }}$ user is expanded as

$$
r_{t}^{(k)}=d_{t}^{(k)}+\sum_{\substack{i=1 \\ i \neq k}}^{K} d_{t}^{(i)} \rho^{(i k)}+n_{t}^{(k)}
$$

where $n_{t}^{(k)}$ is a Gaussian random process with zero mean and variance $\sigma^{2}=N_{0} / 2$. The cross-correlation value $\rho^{(i k)}$ is the $(i, k)^{t h}$ element of the cross-correlation matrix $\mathbf{H}_{t}$.

\section{Iterative CDMA Receiver}

The proposed iterative multi-user CDMA receiver is composed of a metric generator and $K$ single user Turbo decoders. The metric generator uses the outputs of the matched filters, $\mathbf{r}_{t}=\left(r_{t}^{(1)}, \ldots, r_{t}^{(K)}\right)^{\top}$, from the front end of the receiver to generate a metric suitable for use in the $K$ single user Turbo decoders. Each Turbo decoder then uses this metric to improve the bit probabilities, which will be subsequently used as the a-priori input to the metric generator for use in the next iteration. The iterative process continues in this manner until further iterations yield little or no significant improvement.

The main problem of implementing such an iterative multi-user CDMA receiver is that of generating the correct probability values in the metric generator for use in the Turbo decoders. The key point to the proposed iterative algorithm is to find a proper method to update the marginal conditional probability distributions (metric), $p\left(r_{t}^{(k)} \mid d_{t}^{(k)}\right)$, from iteration to iteration. We have explored a variety of methods to extract and update these marginal probabilities $[1,2]$. Two of these receivers only update the probability distribution of the MAI, while another two receivers also include the updating of the probability distributions of the additive correlated Gaussian noise. For the sake of briefness, the current article is limited to the two methods which only update the probability distribution of the MAI. For the iterative multi-user CDMA receiver, each Turbo decoder is modified to produce both uncoded and coded bit probabilities $\operatorname{Pr}\left(b_{r}^{(k)}=b \mid \mathbf{r}^{(k)}\right), b \in\{0,1\}$, and $\operatorname{Pr}\left(d_{t}^{(k)}=d \mid \mathbf{r}^{(k)}\right), d \in\{-1,1\}$, respectively. The metric generator creates the metric $p\left(r_{t}^{(k)} \mid d_{t}^{(k)}\right)$ by assigning the output of the Turbo decoders from iteration $i$ as the $a$ priori input probability for the $i+1$ iteration. For the initial iteration, the metric generator assumes equally likely bit probabilities for all $K$ users. After the required number of iterations have been completed, the systematic bit probabilities $\operatorname{Pr}\left(b_{r}^{(k)}=b \mid \mathbf{r}^{(k)}\right), b \in\{0,1\}$, generated by the $K$ Turbo decoders are used to make the final hard-decision to calculate

$$
\hat{b}_{r}^{(k)}=\operatorname{sign}\left(\frac{\operatorname{Pr}\left(b_{r}^{(k)}=1 \mid \mathbf{r}^{(k)}\right)}{\operatorname{Pr}\left(b_{r}^{(k)}=0 \mid \mathbf{r}^{(k)}\right)}-1\right), \quad \forall k
$$

To reduce the complexity of the derivations for the probability metric, the cross-correlation term $\rho^{(i j)}$ is assumed to be constant and equal to $\rho$ for all $i \neq j, i, j \in$ $\{1, \ldots, K\}$. As a result of this assumption, the discrete cross-correlation matrix $\mathbf{H}_{t}$ becomes a time invariant matrix, represented as $\mathbf{H}$, which has diagonals equal to one and the non-diagonal elements equal to $\rho$. The developed method is, however, general and can be easily modified to incorporate arbitrary correlation values. Note that a similar assumption is used in [9]. The following proposed receivers explore two different methods to update the metric $p\left(r_{t}^{(k)} \mid d_{t}^{(k)}\right)$.

\subsection{Gaussian Approximation of MAI}

In one class of algorithms, the MAI term is approximated by a Gaussian random variable. From (2), the MAI term consists of the sum of $K-1$ discrete binary random variables. From the central limit theorem ${ }^{1}$, as the number of users $K$ increases, the distribution of the MAI term approaches that of a Gaussian. Each single user binary probability distribution consists of the coded bit probabilities $\operatorname{Pr}\left(d_{t}^{(k)}=1\right)$ and $\operatorname{Pr}\left(d_{t}^{(k)}=-1\right), k \in\{1, \ldots, K\}$. The mean and variance of these binary distributions for the $k^{\text {th }}$

\footnotetext{
1 The central limit theorem states that the sum of $n$ statistically independent and identically distributed random variables approach a Gaussian distribution as $n \rightarrow \infty$.
} 
user, $k \in\{1, \ldots, K\}$ are calculated as,

$$
\begin{aligned}
m_{t}^{(k)} & =\operatorname{Pr}\left(d_{t}^{(k)}=1\right)-\operatorname{Pr}\left(d_{t}^{(k)}=-1\right) \\
v_{t}^{2(k)} & =1-\left[\operatorname{Pr}\left(d_{t}^{(k)}=1\right)-\operatorname{Pr}\left(d_{t}^{(k)}=-1\right)\right]^{2}
\end{aligned}
$$

The addition of these binary distributions results in the MAI term $\rho \sum_{\substack{i=1 \\ i \neq k}}^{K} d_{t}^{(i)}$ for the $k^{t h}$ user having a Gaussian distribution with mean and variance:

$$
\begin{aligned}
\mu_{t, M A I}^{(k)} & =\rho \sum_{\substack{i=1 \\
i \neq k}}^{K}\left[\operatorname{Pr}\left(d_{t}^{(i)}=1\right)-\operatorname{Pr}\left(d_{t}^{(i)}=-1\right)\right] \\
& =\rho \sum_{\substack{i=1 \\
i \neq k}}^{K} m_{t}^{(i)} \\
\sigma_{t, M A I}^{2(k)} & =\rho^{2} \sum_{\substack{i=1 \\
i \neq k}}^{K}\left(1-\left[\operatorname{Pr}\left(d_{t}^{(i)}=1\right)-\operatorname{Pr}\left(d_{t}^{(i)}=-1\right)\right]^{2}\right) \\
& =\rho^{2} \sum_{\substack{i=1 \\
i \neq k}}^{K} v_{t}^{2(k)} .
\end{aligned}
$$

The probability distribution $p\left(r_{t}^{(k)} \mid d_{t}^{(k)}=d\right), d \in\{-1,1\}$ for the $k^{\text {th }}$ user is derived as a Gaussian random variable with mean $\mu_{t}^{(k)}=d+\mu_{t, M A I}^{(k)}$ and variance $\sigma_{t}^{2(k)}=\sigma^{2}+$ $\sigma_{t, M A I}^{2(k)}$

\subsection{Discrete Analysis of MAI}

The continuous Gaussian approximation of the MAI receiver becomes accurate when the number of users becomes large. Thus, a different approach is needed to determine the probability distribution of the MAI when the number of users is small. The MAI term $\rho \sum_{\substack{i=1 \\ i \neq k}}^{K} d_{t}^{(i)}$ for user $k$ is a discrete quantity that can only take on $K$ different values since each coded bit $d_{t}^{(i)}$ can only take on values $\{-1,1\}$. As a result, the MAI term can be represented as a random variable with a binomial distribution. This probability distribution is obtained for user $k$ by convolving the other $K-1$ sets of bit probabilities to produce the set of probability values $\left\{P_{t, m}^{(k)}\right\}=\left\{\operatorname{Pr}\left(\sum_{\substack{i=1 \\ i \neq k}}^{K} d_{t}^{(i)}=m\right)\right\}$, where $m \in\{-(K-1),-(K-3), \ldots,(K-3),(K-1)\}$. The value of the metric is determined by convolving the distributions of the MAI and the Gaussian noise. The convolution of these two distributions results in,

$$
\begin{gathered}
p\left(r_{t}^{(k)} \mid d_{t}^{(k)}=d\right)= \\
\frac{1}{\sqrt{2 \pi \sigma^{2}}} \sum_{m} \exp \left[-\frac{\left(r_{t}^{(k)}-d-\rho m\right)^{2}}{2 \sigma^{2}}\right] \times P_{t, m}^{(k)}
\end{gathered}
$$

where $m \in\{-(K-1),-(K-3), \ldots,(K-3),(K-1)\}$ and $d \in\{-1,1\}$.

\section{Numerical Results}

The results presented are obtained by simulating the communication system over an AWGN channel. The simulations are performed using a block size of $M=192 \mathrm{bits}$, $K=5$ users, and spreading signals of $N=7$ chips corresponding to a cross-correlation value of $\rho=1 / 7$. The parameters of $K=5$ users and chip length $N=7$ represent a typically loaded CDMA system. This is the same scenario as used in [9]. The Turbo encoder utilized for simulation is of rate $1 / 3$, consisting of two parallel recursive systematic encoders of 4 memory elements with generator polynomials of $37_{8}$ and $21_{8}$, respectively.

Figure 1 shows the BER performance of the continuous Gaussian approximation of the MAI receiver versus the channel SNR for various values of $\rho$ after the $5^{\text {th }}$ iteration of the multi-user receiver, each with only 1 iteration of the Turbo decoder. The iterations of the overall receiver are referred to as "MUit" in the figures while the iterations for the individual Turbo decoders are referred to as "TDit".

Figures 2 and 3 show the average BER performance of the continuous Gaussian approximation and discrete anal$y$ sis of the MAI receivers after the $5^{\text {th }}$ and $10^{t h}$ system iterations, each with only 1 Turbo decoding iteration.

Figure 4 compares the performance of the two receivers (continuous Gaussian and discrete analysis) after the $5^{\text {th }}$ system iteration, each with only 1 Turbo decoding iteration. The continuous Gaussian approximation and discrete analysis of the MAI receivers give almost similar results with the continuous Gaussian approximation receiver being less complex and only slightly inferior.

Reed et al. [9] derived an iterative multi-user decoder using Turbo Codes for a CDMA system with a set of assumptions on the system similar to the assumptions for the system used in this paper. In [9], simulation results are presented for three multi-user system iterations, each with four Turbo Code iterations and the corresponding average BER performance results are compared with a single-user system using Turbo Code, with four iterations. At an average BER of $10^{-3}$, the Reed et al. iterative multi-user decoder, after three system iterations, is about $0.25 \mathrm{~dB}$ worse than the single-user performance. For the sake of comparison, we obtained results after the third system iteration, each with only one Turbo Code iteration, comparing these results with the single-user system after four Turbo Code iterations. These results are presented in Figure 5. This figure shows that the two decoders result in a similar performance that is about $0.2 \mathrm{~dB}$ worse than the single-user system at an average $B E R$ of $10^{-3}$. The receiver introduced by Reed et al. [9] performs the updating operation of the iterative receiver over a sequence of received bits resulting in an exponential complexity. However, the proposed receivers in this paper perform the updating operation on a 


\begin{tabular}{|c|c|}
\cline { 2 - 2 } \multicolumn{1}{c|}{} & $K=5$ Users \\
\hline Single-User System & 1.00 \\
\hline Continuous Gaussian & 1.51 \\
\hline Discrete Analysis & 1.81 \\
\hline
\end{tabular}

Table 1: Relative Computational Complexity Per User For Continuous Gaussian Approximation and Discrete Analysis of MAI Receivers

per bit basis, resulting in a much lower complexity. Thus, the decoders presented in this paper perform similarly and slightly better at fewer iterations and substantially lower overall complexity as compared to [9].

\section{Complexity Analysis}

The computational complexity of the iterative receivers is linearly dependent on the number of iterations. The continuous Gaussian approximation receiver has a polynomial complexity proportional to $K^{2}$ per iteration ( $K$ is the number of users) while the discrete analysis of the MAI receiver has a polynomial complexity proportional to $K^{3}$ per iteration. Table 1 compares the computational complexity per user of the continuous Gaussian approximation and discrete analysis of the MAI receivers per input bit relative to the single-user Turbo Code system for a fixed number of system iterations $[1,2]$. Table 1 shows that the discrete analysis receiver is $30 \%$ more complex than the continuous Gaussian approximation receiver. This complexity difference is expected since the discrete analysis receiver has to perform the computationally intensive convolution operation. Thus, the continuous Gaussian approximation receiver is the better receiver since it performs similarly to the discrete analysis of the MAI receiver at a smaller computational complexity. The complexity results also show that the continuous Gaussian approximation receiver requires about $50 \%$ more calculations than the single-user case. This is a minimal amount of extra computations for the CDMA multi-user iterative receiver over the single-user system as compared to the multi-user receiver of Reed et al. [9].

\section{References}

[1] D. Stienstra, "Iterative Multi-User Turbo-Code Receiver for DS-CDMA", M.A.Sc. Thesis, E\&CE Dept., Univ. of Waterloo, Jan. 2000.

[2] D. Stienstra and A. K. Khandani, "Iterative Multi-User Turbo-Code Receiver for DS-CDMA," Technical Report UW-E\&CE\#2000-4, Dept. of Elec. and Comp. Eng., University of Waterloo, March 2000.

[3] J. Hagenauer, "Forward Error Correcting for CDMA Systems," IEEE International Symposium on Spread Spectrum Techniques and Applications, Mainz, Germany, pp. 566-569, September 1996.

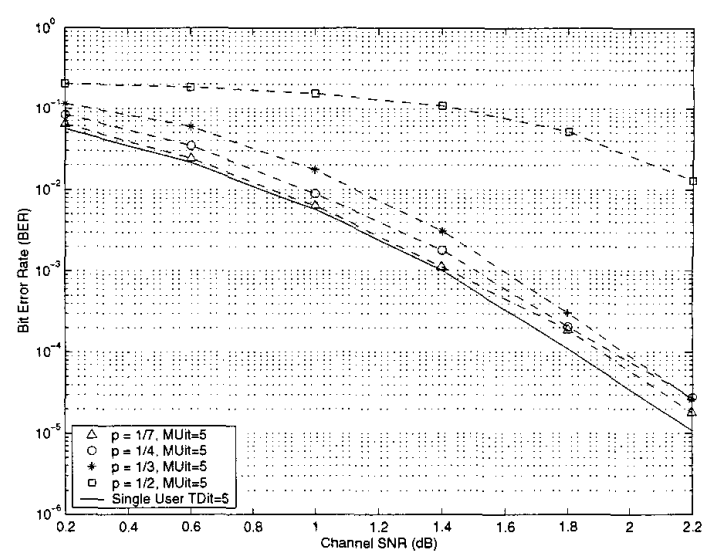

Figure 1: Continuous Approximation of MAI with $K=5$ Users and Block Size $M=192$ for Various Values of $\rho$

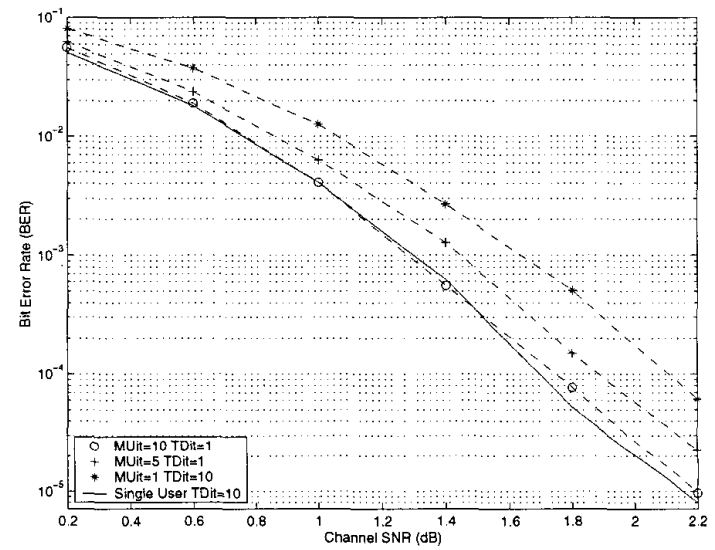

Figure 2: Continuous Approximation of MAI with $K=5$ Users, Block Size $M=192$ and Chip Length $N=7$

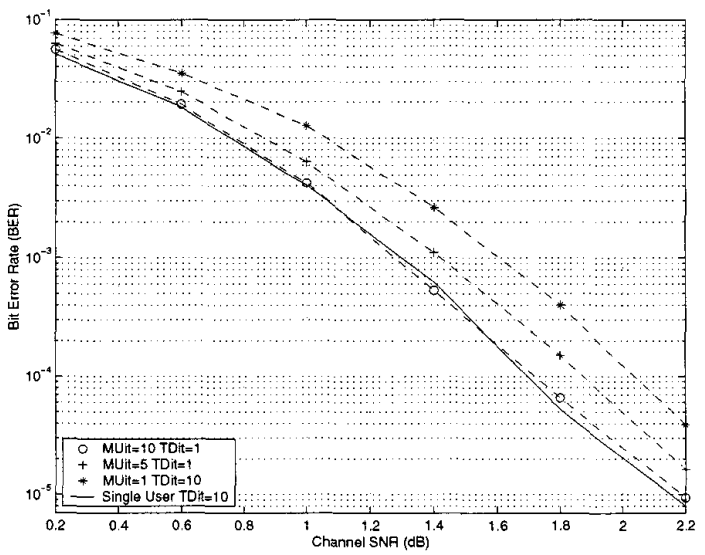

Figure 3: Discrete Analysis of MAI with $K=5$ Users, Block Size $M=192$ and Chip Length $N=7$ 


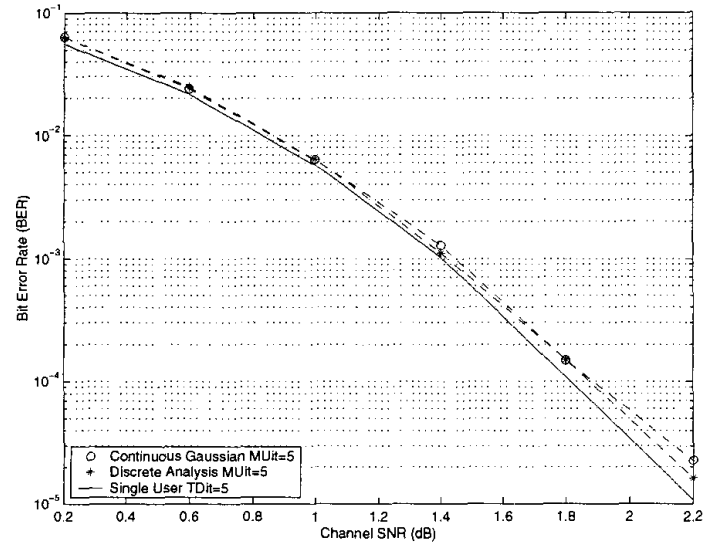

Figure 4: Continuous Approximation Versus Discrete Analysis of MAI with $K=5$ Users, Block Size $M=192$ and Chip Length $N=7$ for 5 iterations

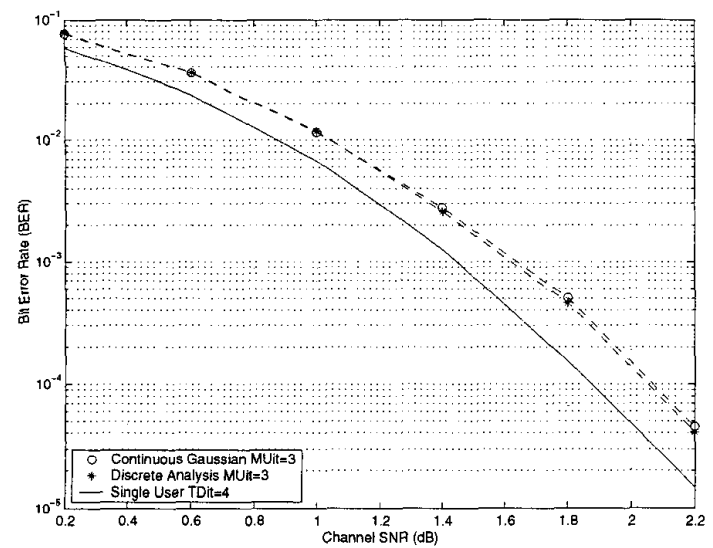

Figure 5: Continuous Approximation Versus Discrete Analysis of MAI with $K=5$ Users, Block Size $M=192$ and Chip Length $N=7$ for 3 iterations
[4] M. I. Moher, "An Iterative Multiuser Decoder for NearCapacity Communications," IEEE Transactions on Communications, vol. 46, pp. 870-880, July 1998.

[5] F. Tarkoy, "Iterative Multi-User Decoding for Asynchronous Users," Proceedings of the IEEE International Symposium on Information Theory, Ulm, Germany, pp. 30, June 1997.

[6] M. C. Reed and P. D. Alexander, "Iterative Multiuser Detection Using Antenna Arrays and FEC on Multi-path Channels," IEEE Journal on Selected Areas in Communications, vol. 17, pp. 2082-2089, December 1999.

[7] M. C. Vanlenti and B. D. Woerner, "Iterative Multiuser Detection for Convolutionally Coded Asynchronous DSCDMA," Proceedings of the IEEE International Symposium on Fersonal, Indoor, and Mobile Radio Communications, Boston, MA, pp. 213-217, 1998.

[8] P. D. Alexander, M. C. Reed, J. A. Asenstorfer, and C. B. Schlegel, "Iterative Multi-User Interference Reduction: Turbo CDMA," IEEE Transactions on Communications, vol. 47, pp. 1008-1014, July 1999.

[9] M. C. Reed, C. B. Schlegel, P. D. Alexander and J. A. Asenstorfer, "Iterative Multiuser Detection for CDMA with FEC: Near-Single-User Performance," IEEE Transactions on Communications, vol. 46, pp. 1693-1699, December 1998.

[10] P. D. Alexander, A. J. Grant, and M. C. Reed, "Iterative Detection In Code-Division Multiple-Access With Error Control Coding," European Transactions on Telecommunications, Special Issue on CDMA Techniques for Wireless Communications Systems, vol.9, no.5, September-October 1998.

[11] X. Wang and H. V. Poor, "Iterative (Turbo) Soft Interference Cancellation and Decoding for Coded CDMA," IEEE Transactions on Communications, vol. 47, pp. 1046-1061, July 1999.

[12] H. El Gamal and E. Gerantiotis, "Iterative Multiuser Detection for Coded CDMA Signals in AWGN and Fading Channels," IEEE Journal on Selected Areas in Communications, vol. 18, pp. 30-41, January 2000.

[13] C. Berrou, A. Glavieux, and P. Thitimajshima, "Near Shannon Limit Error-Correcting Coding and Decoding: TurboCodes (1)," Proceedings IEEE International Conference on Communications, Geneva, Switzerland, pp. 1064-1070, May 1993.

[14] L. R. Bahl, J. Cocke, F. Jelinek, and J. Raviv, "Optimal Decoding of Linear Codes for Minimizing Symbol Error Rate," IEEE Transactions on Information Theory, pp. 284-287, March 1974. 